\title{
Recent Findings on Our Auditory System: It Is Highly Sensitive Owing to the Motility of Sensory Cells
}

\author{
Hiroshi Wada ${ }^{\dagger}$ \\ Department of Bioengineering and Robotics, Toboku University, 6-6-01 Aoba-yama, Sendai, Japan
}

(Received 17 July 2006; accepted 27 April 2007)

Even though the amplitude of tympanic membrane vibrations is only a few nanometres when we speak in a low voice, we can clearly understand what is being said. This is speculated to be due to cochlear amplification. The origin of this amplification is believed to be the motility of mammalian outer hair cells (OHCs), which are located in the organ of Corti of the cochlea. These are the main focuses of this paper. However, firstly, peripheral anatomy is overviewed. Then, the acoustic properties of the outer and middle ears are interpreted. An explanation of the cochlear function follows. Finally, the origin of the motility of OHCs, i.e., the motor protein "prestin," is discussed.

${ }^{\dagger}$ Member of the International Institute of Acoustics and Vibration (IIAV)

\section{INTRODUCTION}

The ears are paired sense organs, which collect, transmit, and detect acoustic impulses. Each of them is comprised of three main parts: the outer ear, middle ear, and inner ear. Travelling sound is focused into the external auditory canal by the pinna, causing vibration of the tympanic membrane and motion of the three ossicles in the middle ear. Their motion is transmitted to the cochlea of the inner ear. The mechanical motion of the basilar membrane in the organ of Corti of the cochlea is then transduced into encoded nerve signals in the cochlea, which are transmitted to the brain.

Even though the amplitude of the displacement of tympanic membrane vibrations is only a few nanometres when we speak in a low voice, we can clearly understand what is being said. This is thought to be aided by cochlear amplification caused by the motility of outer hair cells $(\mathrm{OHCs})$, which are located in the organ of Corti. The origin of this motility is believed to be associated with a membrane protein in the lateral wall of OHCs. The gene that codes for this protein has been identified and termed "prestin." Prestin has been found to be a direct voltage-to-force converter, which can operate at microsecond rates.

First in this paper, actual measurement results of the tympanic membrane vibrations are shown. Second, the dynamic behaviour of the middle ear and the cochlea is presented. Third, the motility of the isolated OHC is demonstrated, and the function of the OHCs, which behave like actuators of mechanical structures, is discussed. Finally, images of prestin, obtained by an atomic force microscope, are displayed.

\section{REMARKABLE SENSITIVITY OF THE EAR}

Sound is energy that is transmitted by pressure waves in air and is the objective cause of the sensation of hearing. Figure 1 shows the auditory response area for humans. ${ }^{1,2}$ The area is bounded on one side by the threshold of pain and on the other by the threshold of audibility as a function of fre- quency. The difference between these thresholds, i.e., the dynamic range, is quite wide, nearly $130 \mathrm{~dB}$ at a frequency of $4.0 \mathrm{kHz}$. High-end recording equipment has a dynamic range of $90 \mathrm{~dB}$. This means that our ears have greater dynamic range than recorded sound.

\section{OVERVIEW OF PERIPHERAL ANATOMY: OUTER, MIDDLE, AND INNER EARS}

Figure 2 displays a computer-aided reconstruction of the human middle and inner ears, which was obtained from a fixed temporal bone extracted from a fresh cadaver. The relationship of size and location among the various components of the peripheral auditory system can be clearly understood. In humans, as shown in Fig. 3, the external auditory canal with a diameter of $7 \mathrm{~mm}$ and a length of $30 \mathrm{~mm}$, which is slightly bent and elliptical, is terminated by a conical-shaped tympanic membrane with a diameter of $10 \mathrm{~mm}$ and a thickness of $0.1 \mathrm{~mm}$. Three ossicles, namely the malleus, incus, and stapes, are located in the tympanic cavity. The malleus is attached to the tympanic membrane, the incus lies between the malleus and the stapes, and the stapes is connected to the cochlea.

Figure 4 depicts the human cochlea with a length of $35 \mathrm{~mm}$, which is spiral-shaped and has three fluid-filled compartments, i.e., the scala vestibuli, the scale media, and the scala tympani. They are separated by Reissner's membrane and the basilar membrane. The scala vestibuli and scala tympani contain perilymph, and the scala media contains endolymph. At the basal end, the scala vestibuli has an oval window and the scala tympani has a round window. The base of the stapes, called the footplate, is sealed by a flexible ligament and transmits the vibration of the middle ear to the fluid in the scala vestibuli.

As shown in Fig. 5, the organ of Corti sits on the basilar membrane and contains two types of hair cells, i.e., the inner hair cells (IHCs) and the OHCs. There are approximately $3,500 \mathrm{IHCs}$ and $12,000 \mathrm{OHCs}$ in humans. ${ }^{3}$ Hairlike structures, i.e., stereocilia, extend from the top of these cells. The organ 